\title{
Existe diferença no posicionamento dos eletrodos da TENS no tratamento da dismenorreia primária? Estudo randomizado
}

\author{
Is there a difference in the positioning of \\ TENS electrodes in the treatment of primary \\ dysmenorrhea? Randomized study
}

\author{
Amanda da Rocha Rodrigues ${ }^{1}$ (i) \\ Fernanda de Oliveira Almeida² (1) \\ Priscila de Oliveira Januário 3 (1) \\ Ariela Torres $\mathrm{Cruz}^{4}$ (D)
}

${ }^{1}$ Autora para correspondência. Centro Universitário de Barra Mansa (Barra Mansa). Rio de Janeiro, Brasil. rochaamanda.r@gmail.com ${ }^{2-4}$ Centro Universitário de Barra Mansa (Barra Mansa). Rio de Janeiro, Brasil. nanda03.11@hotmail.com, pri.januario@gmail.com, ariela_tcruz@yahoo.com.br

RESUMO | INTRODUÇãO: Dismenorreia é a condição dolorosa mais frequente em adolescentes e mulheres jovens causando absenteísmo e presenteísmo no trabalho e na escola. É caracterizada por um quadro álgico leve, moderado ou severo na região pélvica anterior do tipo cólica, o qual pode acontecer antes, durante ou depois do fluxo menstrual. OBJETIVO: Comparar a influência da Estimulação Elétrica Nervosa Transcutânea (TENS) na dor pélvica causada pela dismenorreia primária com os eletrodos aplicados na região pélvica anterior e posterior. MÉTODOS: 50 universitárias foram aleatoriamente distribuídas em dois grupos de 25 voluntárias: Grupo Região Pélvica Anterior (GA) e Grupo Região Pélvica Posterior (GP), que foram submetidas a TENS durante 30 minutos tendo a intensidade aumentada a cada 10 minutos e avaliadas pela Escala Visual Analógica de Dor antes, depois e duas horas após o término do tratamento. As participantes do GA tiveram os eletrodos aplicados na região pélvica anterior e as do GB na região pélvica posterior. RESULTADOS: Houve uma diminuição do quadro álgico nos momentos antes e após o tratamento (GA e GP p <0,0001) e antes e duas horas após o tratamento (GA e GP $p<0,0001$ ). Nos momentos depois do tratamento e duas horas após o seu término foi possível observar aumento do quadro álgico no $G A(p=1,0000)$ e diminuição no $\mathrm{GP}$, porém os valores não foram estatisticamente significativos ( $p=0,8443$ ). CONCLUSÃO: $O$ uso da TENS contribuiu para a redução do quadro álgico das mulheres de ambos os grupos, sem diferença estatística entre estes. Registro Brasileiro de Ensaios Clínicos: RBR-67cjv5.

PALAVRAS-CHAVE: Dismenorreia. Estimulação Elétrica Nervosa Transcutânea. Fisioterapia.
ABSTRACT | INTRODUCTION: Dysmenorrhea is the most frequent painful condition in adolescents and young women that causes absenteeism and presenteeism at work and school. It is characterized by a mild, moderate, or severe pain in the anterior pelvic region of the colic type, which can happen before, during, or after menstrual flow. OBJECTIVE: To compare the influence of Transcutaneous Electrical Nerve Stimulation (TENS) in pelvic pain caused by primary dysmenorrhea with the electrodes applied in the anterior and posterior pelvic region. METHODS: 50 university students were randomly assigned to two groups of 25 volunteers: Anterior Pelvic Region Group (GA) and Posterior Pelvic Region Group (GP), who were submitted to TENS for 30 minutes and the intensity increased every 10 minutes and evaluated by the Visual Analog Pain Scale before, after and two hours after the end of treatment. GA participants had the electrodes applied in the anterior pelvic region and GB in the posterior pelvic region. RESULTS: There was a decrease in the pain in the moments before and after treatment (GA and GP $p<0.0001$ ) and before and two hours after treatment (GA and GP $p<0.0001$ ). In the moments after the treatment and two hours after its end, it was possible to observe an increase in the pain in GA $(p=1.0000)$ and a decrease in the GP, however, the values were not statistically significant $(p=0.8443)$. CONCLUSION: The use of TENS contributed to the reduction of pain in women in both groups, without statistical difference between them. Brazilian Registry of Clinical Trials: RBR-67cjv5.

KEYWORDS: Dysmenorrhea. Transcutaneous Electrical Nerve Stimulation. Physical Therapy Specialty. 


\section{Introdução}

A dismenorreia é uma condição dolorosa que acomete de $50 \%$ a $90 \%$ das mulheres em idade reprodutiva, sendo mais frequente em adolescentes e mulheres jovens. É o sintoma mais comum em consultas ginecológicas caracterizada por um quadro álgico severo comumente do tipo cólica, sendo classificada de acordo com sua intensidade podendo ser leve, moderada ou severa, a qual antecede ou acompanha o fluxo menstrual ${ }^{1}$.

A partir de seu aspecto clínico, existem dois tipos de dismenorreia: a primária, chamada de funcional, ou espasmódica, associada com períodos menstruais ovulatórios normais, e a secundária, também conhecida como orgânica que pode surgir anos após o início da menstruação e está relacionada com doenças uterinas ${ }^{2}$.

O início da dismenorreia primária ocorre normalmente 6 a 24 meses após a menarca, quando os ciclos ovulatórios são determinados ${ }^{1}$. Esta ocorre em pacientes com ausência de lesões orgânicas, não apresentando etiologia bem definida. Dentre as causas, evidenciam-se a psicogênica, a miometrial, a endócrina e a influência das prostaglandinas e leucotrienos. Acredita-se que a maior liberação de prostaglandinas no fluxo menstrual causa contração uterina e dor ${ }^{3}$.

Os sintomas da dismenorreia primária são dor na região pélvica anterior (baixo ventre) e região pélvica posterior (região lombar), podendo irradiar para membros inferiores. Pode também estar acompanhada de náusea, cefaleia, irritabilidade, diarreia ou constipação, vômitos, vertigens e sensibilidade mamária. A gravidade pode estar associada à menarca antecipada, duração do fluxo menstrual, obesidade, história de abuso sexual, tabagismo, etilismo e distúrbios emocionais?.

Frequentemente a dismenorreia é sub-diagnosticada e subtratada. Os principais tratamentos incluem medicamentos como anti-inflamatórios não esteroides, analgésicos e pílulas anticoncepcionais orais. Estes apresentam efeitos colaterais como ulceração, intolerância gastrointestinal, inibição de agregação plaquetária, da motilidade uterina, da função renal por intermédio de prostaglandina e alterações de sensibilidade. Embora sejam pouco estudadas, as terapias alternativas são frequentemente usadas por proporcionar melhor controle dos sintomas e apresentarem poucos efeitos colaterais. Os dados ainda são limitados e contraditórios sobre a eficácia de modificações na dieta, utilização de suplementos e ervas ${ }^{1 / 4}$. Outros tratamentos não farmacológicos incluem psicoterapia e fisioterapia, tornando-se esta de extrema relevância e eficiência em mulheres que não podem fazer o tratamento farmacológico convencional ${ }^{5}$.

A fisioterapia apresenta recursos terapêuticos para diminuir o desconforto e contribuir na prevenção da dismenorreia primária com técnicas como alongamento global, liberação miofascial, massoterapia, contração perineal, ondas curtas, manipulação de vértebras, ultrassom pulsado e estimulação elétrica nervosa transcutânea (TENS) ${ }^{6}$.

A TENS é um recurso não invasivo que vem sendo amplamente recomendado para o tratamento da dismenorreia primária para aliviar a dor pélvica. É um método de estimulação dos nervos periféricos através de eletrodos acoplados à pele, que atua nos sistemas moduladores da dor, aumentando a tolerância à dor e causando analgesia. Seu efeito é baseado na teoria das comportas e na ativação do sistema de opiódes endógenos ${ }^{7}$.

Estudos mostram a utilização da TENS como recurso terapêutico no tratamento da dismenorreia primária, contudo, as pesquisas não padronizam a localização dos eletrodos, assim como os outros parâmetros utilizados ${ }^{8-11}$. A aplicação dos eletrodos na região pélvica anterior estimula os nervos sensoriais de $T 12$, raiz das fibras sensoriais uterinas. A aplicação de eletrodos na região pélvica posterior apresenta proximidade do plexo hipogástrico superior, que transmite os impulsos dolorosos viscerais do útero ${ }^{7}$. Esses achados relacionam-se à maior inervação do útero ocorrer nos segmentos da transição toracolombar e segmentos sacrais associando esses dados com a elasticidade do tecido conetivo, que estaria aumentada em função da variação hormonal que ocorrem nos ciclos menstruais ${ }^{12}$. Portanto, torna-se fundamental o desenvolvimento de estudos que abordem recursos fisioterápicos de baixo custo e sem efeitos colaterais que possam aliviar a dor pélvica decorrente da dismenorreia primária. Com base no assunto, este estudo teve como objetivo comparar a influência da TENS na dor pélvica causada pela dismenorreia primária com os eletrodos aplicados na região pélvica anterior e posterior, tendo a hipótese que a aplicação dos eletrodos na região pélvica posterior é mais eficaz que aplicação dos eletrodos na região pélvica anterior. 


\section{Métodos}

Trata-se de um estudo clínico randomizado que teve início após a aprovação do Comitê de Ética em Pesquisa do Centro Universitário de Barra Mansa (UBM), sob parecer número 2.764.865 (CAAE 91396518.0.0000.5236), respeitando todos os princípios éticos que norteiam a pesquisa, bem como a privacidade de seus conteúdos, como preconizam os documentos internacionais e a Resolução 466/12 do Conselho Nacional de Saúde do Ministério da Saúde, sendo inscrito no Registro Brasileiro de Ensaios clínicos: RBR-67cjv5.

A seleção da amostra foi realizada por conveniência, utilizando a amostragem não probabilística. Participaram 50 universitárias, com idade entre 18 e 30 anos, que aceitaram participar voluntariamente do estudo após divulgação e convite realizados em salas de aula de um Centro Universitário no interior do estado do Rio de Janeiro, conforme o Termo de Consentimento Livre e Esclarecido (TCLE). O recrutamento foi realizado em julho de 2018 , e o tratamento foi realizado entre os meses de julho a setembro de 2018. Foram incluídas no estudo as voluntárias com idade entre 18 a 30 anos, com dor pélvica ocasionada por dismenorreia primária, que estavam entre 0 primeiro e o terceiro dia do ciclo menstrual e que apresentaram graus entre 1 e 10 na Escala Visual Analógica de dor (EVA). Foram excluídas aquelas que estavam utilizando analgésicos e outras terapias para a dor pélvica, que apresentaram doenças ginecológicas e dores abdominais não diagnosticadas. Foram excluídas também as voluntárias com marca-passo cardíaco, complicações cardíacas, e as que apresentaram grau 0 na EVA. A randomização foi realizada por sorteio, utilizando um envelope opaco que continha dois outros envelopes selados, descritos os tratamentos propostos. As participantes souberam a que tratamento seriam submetidas quando chegaram para realizar o tratamento. A avaliadora treinada foi a mesma que selecionou, realizou o sorteio, as avaliou e tratou. As participantes foram distribuídas em dois grupos iguais de 25 voluntárias: Grupo região pélvica anterior (GA) e Grupo região pélvica posterior (GP).

Os objetivos e as condutas do estudo foram apresentados às participantes. Primeiramente, elas responderam a um questionário elaborado pelas autoras para traçar o perfil sociodemográfico e de saúde das mesmas, e verificar as que se enquadravam nos critérios de inclusão e exclusão. Esse questionário foi aplicado por uma única pesquisadora antes do procedimento. Assim que os primeiros sintomas apareceram, as voluntárias entraram em contato com as pesquisadoras para que $o$ atendimento fosse agendado o mais rápido possível. As participantes foram avaliadas por meio da EVA antes da aplicação da TENS, depois de sua aplicação e 2 horas após o seu término. Essa é uma escala de 0 a 10, sendo que 0 significa ausência total de dor e 10 o nível de dor máxima suportável pela paciente. Foram consideradas dor leve de 1 a 3, média de 4 a 7 e máxima de 8 a 10.

Todas as participantes foram submetidas a um atendimento utilizando-se o aparelho de TENS Neurodyn III da marca (IBRAMED ${ }^{\circledR}$ - Brasil, 2018) de 2 canais. Foram usados 4 eletrodos de borracha impregnada por carbono com medidas $5 \times 5 \mathrm{~cm}$, que foram devidamente acoplados à pele por gel condutor e fixados com fita crepe, localizados na região pélvica em fogo cruzado. Foi realizada assepsia do local da aplicação dos eletrodos com álcool $70 \%$ e algodão. Foram utilizados os seguintes parâmetros: frequência de $10 \mathrm{Hertz}(\mathrm{Hz})$, duração de pulso $300 \mu$ s durante 30 minutos, e a intensidade era aumentada a cada 10 minutos. A intensidade inicial era a máxima que a participante considerasse como confortável. As participantes do GA permaneceram em decúbito dorsal, e os eletrodos aplicados na região pélvica anterior ( 1 eletrodo a $2 \mathrm{~cm}$ medialmente da espinha ilíaca ântero superior direita, 1 eletrodo a 2 $\mathrm{cm}$ medialmente da espinha ilíaca ântero superior esquerda e 2 eletrodos, sendo cada um $2 \mathrm{~cm}$ abaixo dos eletrodos aplicados superiormente), e as participantes do GP permaneceram em decúbito ventral, com um travesseiro debaixo do abdômen para evitar uma hiperlordose lombar, e os eletrodos foram aplicados na região pélvica posterior (2 eletrodos a $2 \mathrm{~cm}$ acima das espinhas ilíacas póstero superiores, sendo um a direita e outro à esquerda, e 2 eletrodos a $2 \mathrm{~cm}$ abaixo das espinhas ilíacas póstero superiores, sendo um a direita e outro à esquerda).

Os dados coletados seguiram distribuição normal, portanto, o perfil sociodemográfico e de saúde dos grupos de estudo verificados através do questionário realizado no primeiro contato com as participantes foram expressos pela frequência absoluta e relativa, média e desvio padrão, considerando o intervalo de confiança (IC) de 95\%. Na comparação das variáveis entre grupos, foram preferidos os testes t-Student ou Mann- Whitney para duas categorias e ANOVA ou 
Kruskal-Wallis com post hoc de Dunn para mais de duas categorias com nível de significância de 5\%. Em relação a análise dos tempos de tratamento, foram realizados os testes ANOVA One-way e Kruskal-Wallis com post hoc de Tuckey e nível de significância de 5\%. Para os testes estatísticos foi utilizado o programa BioEstat versão 5.0.

\section{Resultados}

Foram entrevistadas 165 voluntárias. Destas, foram excluídas 108 por só responderem o contato inicial, 4 por apresentarem ovário policístico sem tratamento e 3 por apresentarem dor abdominal não diagnosticada (Fluxograma 1). O perfil das participantes encontra-se na Tabela 1. Após análise dos tempos antes e depois do tratamento no GA, foi possível observar que houve redução estatisticamente significativa do quadro álgico das participantes do estudo ( $p=0,0001$ ). Já entre os tempos depois do tratamento e duas horas após o término do mesmo, houve um aumento do quadro álgico $(\mathrm{p}=0,7700)$, porém, verificando os valores antes do tratamento, e duas horas após o seu término, observou-se que houve uma diminuição estatisticamente significativa do quadro álgico ( $p<0,0001)$. No GP, comparando os tempos antes e depois do tratamento, houve redução estatisticamente significativa do quadro álgico $(p<0,0001)$, assim como nos tempos antes e duas horas após o seu término $(p<0,0001)$. Já entre os tempos depois do tratamento e duas horas após o término do mesmo, houve redução da dor, mas não foi estatisticamente significativo ( $p=0,5259)$. Ao comparar o GA e GP nos tempos antes $(p=0,6578)$, depois do tratamento $(p=0,8080)$ e duas horas após o seu término $(p=0,4141)$, observou-se que não houve diferença estatisticamente significativa (Gráfico 1).

Fluxograma 1. Desenho de estudo e acompanhamento das participantes durante o ensaio

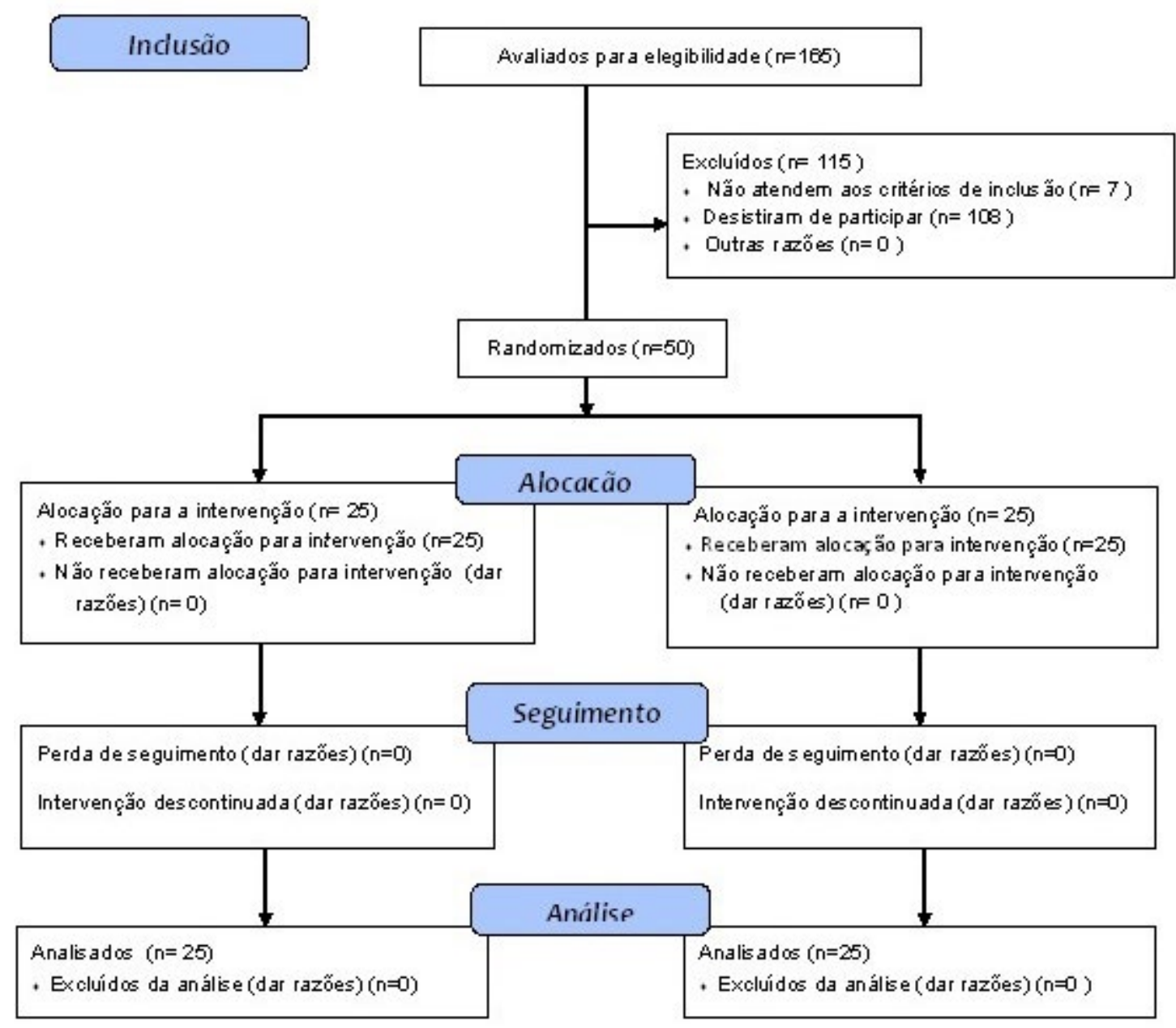


Tabela 1. Perfil dos grupos de estudo (continua)

\begin{tabular}{|c|c|c|c|c|c|c|c|}
\hline \multicolumn{4}{|c|}{ GA } & \multicolumn{4}{|c|}{ GB } \\
\hline $\begin{array}{l}\text { Variáveis } \\
\text { Idade }\end{array}$ & n (\%) & Média/DP & IC & n (\%) & Média/DP & IC & Comparação P-valor \\
\hline 18 a 20 anos & $9(36)$ & $5 \pm 2,73$ & $2,90-7,09$ & $14(56)$ & $7,5 \pm 4,18$ & $5,08-9,91$ & $\mathrm{~ns}^{3}$ \\
\hline 21 a 25 anos & $13(52)$ & $7 \pm 3,89$ & $4,64-9,35$ & $9(36)$ & $5 \pm 2,73$ & $2,90-7,09$ & $\mathrm{~ns}^{3}$ \\
\hline 26 a 30 anos & $3(12)$ & $6,5 \pm 3,60$ & $2,44-15,44$ & $2(8)$ & $1,5 \pm 0,70$ & $4,78-7,78$ & $0,317^{2}$ \\
\hline \multicolumn{8}{|c|}{ Qual idade ocorreu a menarca? } \\
\hline$<10$ anos & $6(24)$ & $3,5 \pm 1,87$ & $1,53-5,46$ & $2(8)$ & $1,5 \pm 0,70$ & $4,78-7,78$ & $0,027^{*}$ \\
\hline 11 a 15 anos & $19(76)$ & $10 \pm 5,62$ & $7,29-12,70$ & $23(92)$ & $12 \pm 6,78$ & $9,06-14,93$ & $0,156^{1}$ \\
\hline \multicolumn{8}{|l|}{ Ciclo Menstrual } \\
\hline Regular & $14(56)$ & $7,5 \pm 4,18$ & $5,08-9,91$ & $18(72)$ & $9,5 \pm 5,33$ & $6,84-12,15$ & $0,127^{2}$ \\
\hline $\begin{array}{l}\text { Irregular } \\
\text { Fluxo }\end{array}$ & $11(44)$ & $6 \pm 3,31$ & $3,77-8,22$ & $7(28)$ & $4 \pm 2,16$ & $2,00-5,99$ & $0,074^{2}$ \\
\hline Moderado & $20(80)$ & $10,5 \pm 5,91$ & $7,73-13,26$ & $17(68)$ & $9 \pm 5,04$ & $6,40-11,59$ & $0,208^{2}$ \\
\hline Severo & $5(20)$ & $3 \pm 1,58$ & $1,03-4,96$ & $8(32)$ & $4,5 \pm 2,44$ & $2,45-6,54$ & $0,103^{2}$ \\
\hline \multicolumn{8}{|c|}{ Em qual região se localiza a dor? } \\
\hline Lombo-Sacral & $2(8)$ & $1,5 \pm 0,70$ & $4,78-7,78$ & $4(16)$ & $2,5 \pm 1,29$ & $0,44-4,55$ & $0,130^{2}$ \\
\hline Pelve & $12(48)$ & $6,5 \pm 3,60$ & $4,21-8,78$ & $9(36)$ & $5 \pm 2,73$ & $2,90-7,09$ & $0,148^{2}$ \\
\hline Membros Inf. & $1(4)$ & $1 \pm 1$ & $1-1$ & $0(0)$ & $0 \pm 0$ & $0-0$ & $0,438^{2}$ \\
\hline Todas Altern. & $8(32)$ & $4,5 \pm 2,44$ & $2,45-6,54$ & $3(12)$ & $2 \pm 1$ & $2,44-15,44$ & $<0,05^{3^{*}}$ \\
\hline Lombo-Sacral+ & $1(4)^{\prime}$ & $1 \pm 1$ & $1-1$ & $7(28)$ & $4 \pm 2,16$ & $2,00-5,99$ & $<0,05^{3^{*}}$ \\
\hline Pelve & & & & & & & \\
\hline $\begin{array}{l}\text { Lombo-Sacral+ } \\
\text { Membro Inf. }\end{array}$ & $1(4)$ & $1 \pm 1$ & $1-1$ & $2(8)$ & $1,5 \pm 0,70$ & $4,78-7,78$ & $0,317^{2}$ \\
\hline \multicolumn{8}{|l|}{ Qual tipo da dor? } \\
\hline Cólica & $11(44)$ & $6 \pm 3,31$ & $3,77-8,22$ & $5(20)$ & $3 \pm 1,58$ & $1,03-4,96$ & $<0,05^{3^{*}}$ \\
\hline Latejante & $4(16)$ & $2,5 \pm 1,29$ & $0,44-4,55$ & $6(24)$ & $3,5 \pm 1,87$ & $1,53-5,46$ & $0,139^{2}$ \\
\hline Pontada & $5(20)$ & $3 \pm 1,58$ & $1,03-4,96$ & $7(28)$ & $4 \pm 2,16$ & $2,00-5,99$ & $0,142^{2}$ \\
\hline Todas as altern. & $2(8)$ & $1,5 \pm 0,70$ & $4,78-7,78$ & $0(0)$ & $0 \pm 0$ & $0-0$ & $0,102^{2}$ \\
\hline Latejante+ & $2(8)$ & $1,5 \pm 0,70$ & $4,78-7,78$ & $1(4)$ & $1 \pm 1$ & $1-1$ & $0,317^{2}$ \\
\hline Pontada & & & & & & & \\
\hline Cólica+ Latejante & $1(4)$ & $1 \pm 1$ & $1-1$ & $2(8)$ & $1,5 \pm 0,70$ & $4,78-7,78$ & $0,317^{2}$ \\
\hline Cólica +Pontada & $0(0)$ & $0 \pm 0$ & $0-0$ & $4(16)$ & $2,5 \pm 1,29$ & $0,44-4,55$ & $<0,05^{3^{*}}$ \\
\hline
\end{tabular}


Tabela 1. Perfil dos grupos de estudo (conclusão)

\begin{tabular}{|c|c|c|c|c|c|c|c|}
\hline \multicolumn{8}{|c|}{ Em qual dia do ciclo a dor é mais intensa? } \\
\hline $1^{\circ} \mathrm{dia}$ & $6(24)$ & $3,5 \pm 1,87$ & $1,53-5,46$ & 14(56) & $7,5 \pm 4,18$ & $5,08-9,91$ & $<0,05^{3 *}$ \\
\hline $2^{\circ}$ dia & $2(8)$ & $1,5 \pm 0,70$ & $4,78-7,78$ & $1(4)$ & $1 \pm 1$ & $1-1$ & $0,317^{2}$ \\
\hline $3^{\circ} \mathrm{dia}$ & $4(16)$ & $2,5 \pm 1,29$ & $0,44-4,55$ & $0(0)$ & $0 \pm 0$ & $0-0$ & $<0,05^{3^{*}}$ \\
\hline Todas as altern. & $5(20)$ & $3 \pm 1,58$ & $1,03-4,96$ & $4(16)$ & $2,5 \pm 1,29$ & $0,44-4,55$ & $0,317^{2}$ \\
\hline $1^{\circ} \mathrm{e} 2^{\circ} \mathrm{dia}$ & $7(28)$ & $4 \pm 2,16$ & $2,00-5,99$ & $4(16)$ & $2,5 \pm 1,29$ & $0,44-4,55$ & $0,061^{2}$ \\
\hline $2^{\circ} \mathrm{e} 3^{\circ} \mathrm{dia}$ & $1(4)$ & $1 \pm 1$ & $1-1$ & $2(8)$ & $1,5 \pm 0,70$ & $4,78-7,78$ & $0,317^{2}$ \\
\hline \multicolumn{8}{|c|}{ A dor ocorre fora do período menstrual? } \\
\hline Sim & $12(48)$ & $6,5 \pm 3,60$ & $4,21-8,78$ & $5(20)$ & $3 \pm 1,58$ & $1,03-4,96$ & $0,007^{1 *}$ \\
\hline Não & $13(52)$ & $7 \pm 3,89$ & $4,64-9,35$ & $20(80)$ & $10,5 \pm 5,91$ & $7,73-13,26$ & $0,029^{1 *}$ \\
\hline \multicolumn{8}{|c|}{ Por quanto tempo essa dor persiste? } \\
\hline 8 horas & $6(24)$ & $3,5 \pm 1,87$ & $1,53-5,46$ & $11(44)$ & $6 \pm 3,31$ & $3,77-8,22$ & $<0,05^{3^{*}}$ \\
\hline 12 horas & $2(8)$ & $1,5 \pm 0,70$ & $4,78-7,78$ & $3(12)$ & $6,5 \pm 3,60$ & $2,44-15,44$ & $0,317^{2}$ \\
\hline 24 horas & $14(56)$ & $7,5 \pm 4,18$ & $5,08-9,91$ & $5(20)$ & $3 \pm 1,58$ & $1,03-4,96$ & $<0,05^{3^{*}}$ \\
\hline 72 horas & $3(12)$ & $6,5 \pm 3,60$ & $2,44-15,44$ & $6(24)$ & $3,5 \pm 1,87$ & $1,53-5,46$ & $0,058^{2^{*}}$ \\
\hline \multicolumn{8}{|c|}{ Sintomas mais citados } \\
\hline Irritabilidade & $17(28)$ & $9 \pm 5,04$ & $6,4011,59$ & $14(22)$ & $7,5 \pm 4,18$ & $5,08-9,91$ & $0,074^{2}$ \\
\hline Cefaleia & $17(28)$ & $9 \pm 5,04$ & $6,4011,59$ & $14(22)$ & $7,5 \pm 4,18$ & $5,08-9,91$ & $0,074^{2}$ \\
\hline $\begin{array}{l}\text { Sensação de } \\
\text { Inchaço }\end{array}$ & $16(26)$ & $8,5 \pm 4,76$ & $5,96-11,03$ & $20(32)$ & $10,5 \pm 5,91$ & $7,73-13,26$ & $<0,05^{3^{*}}$ \\
\hline Diarreia & $6(10)$ & $3,5 \pm 1,87$ & $1,53-5,46$ & $6(10)$ & $3,5 \pm 1,87$ & $1,53-5,46$ & \\
\hline Sudorese & $5(8)$ & $3 \pm 1,58$ & $1,03-4,96$ & $9(14)$ & $5 \pm 2,73$ & $2,90-7,09$ & $<0,05^{3^{*}}$ \\
\hline \multicolumn{8}{|c|}{ Costuma usar analgésicos para aliviar os sintomas da dismenorreia? } \\
\hline Sim & $6(24)$ & $3,5 \pm 1,87$ & $1,53-5,46$ & $4(16)$ & $2,5 \pm 1,29$ & $0,44-4,55$ & $0,168^{1}$ \\
\hline Não & $19(76)$ & $10 \pm 5,62$ & $7,29-12,70$ & $21(84)$ & $11 \pm 6,20$ & $8,17-13,82$ & $0,298^{1}$ \\
\hline \multicolumn{8}{|c|}{ Utiliza contraceptivo? } \\
\hline Sim & $10(40)$ & $5,5 \pm 3,02$ & $3,33-7,66$ & 12(48) & $6,5 \pm 3,60$ & $4,21-8,78$ & $0,244^{1}$ \\
\hline Não & $15(60)$ & $8 \pm 4,47$ & $5,52-10,47$ & $13(53)$ & $7 \pm 3,89$ & $4,64-9,35$ & $0,266^{1}$ \\
\hline \multicolumn{8}{|c|}{ Tem conhecimento da fisioterapia no tratamento da dismenorreia? } \\
\hline Sim & $10(40)$ & $5,5 \pm 3,02$ & $3,33-7,66$ & $4(16)$ & $2,5 \pm 1,29$ & $0,44-4,55$ & $0,011^{1 *}$ \\
\hline Não & $15(60)$ & $8 \pm 4,47$ & $5,52-10,47$ & $21(84)$ & $11 \pm 6,20$ & $8,17-13,82$ & $0,056^{1 *}$ \\
\hline
\end{tabular}

Legenda: Aspectos sociodemográficos e de saúde expressos em frequência absoluta e relativa- $\mathrm{n}$ (\%), média e desvio padrão- DP; GA- grupo região pélvica anterior; GP- grupo região pélvica posterior; ns- não significativo; ${ }^{1}$ Teste t-Student ou Mann- Whitney; ${ }^{2}$ ANOVA ou Kruskal-Wallis com post hoc de ${ }^{3}$ Dunn; *nível de significância de 5\%; IC-Intervalo de confiança $95 \%$. 
Gráfico 1. Comparação entre momentos do GA e GP

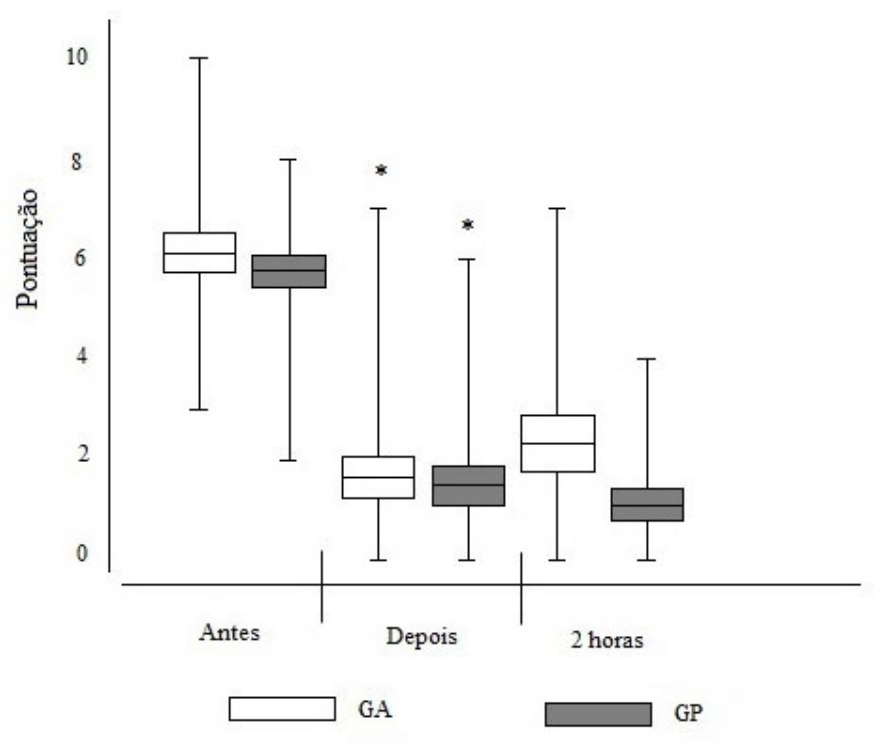

Comparação dos valores de EVA antes, depois e duas horas após o término do tratamento, tanto do GA quanto do GP.

\section{Discussão}

O não cegamento da avaliação e randomização é considerado como uma das limitações deste estudo, assim como o tamanho da amostra. Como utilizou-se a amostragem não probabilística, não foi realizado o cálculo amostral. O número reduzido permite considerar os resultados apenas para população em questão. Porém, é um estudo exploratório com importante implicação prática, podendo esses achados contribuir para uma tomada de decisão dos fisioterapeutas durante o processo de tratamento.

Segundo Silva et al.², inúmeras pesquisas demonstram o predomínio da dismenorreia primária na população jovem adulta, entre 16 e 43 anos, dado confirmado por Silva et al. $\frac{13}{13}$ que entrevistaram 107 voluntárias entre 18 e 45 anos com média de idade de 31,5 $\pm 7,6$ anos. Na atual pesquisa, todas as voluntárias apresentaram entre 18 e 30 anos, com média de idade de $21,66 \pm 2,5$ anos. A literatura também mostra que a maior incidência de dismenorreia é em mulheres com a menarca por volta dos 12 anos de idade ${ }^{14}$. No presente estudo, observou-se que $84 \%$ das participantes afirmaram que sua menarca ocorreu entre 11 e 15 anos.

Um histórico de ciclo menstrual irregular é descrito como fator de risco determinante da dismenorreia. As muIheres que apresentam ciclo menstrual irregular mensalmente obterão quase duas vezes mais probabilidade de desenvolver a dismenorreia em comparação com aquelas que têm o ciclo regular ${ }^{15}$. Na presente pesquisa, a maioria das participantes de ambos os grupos apresentaram ciclo menstrual regular (GA 56\% e GP 72\%) e dor menstrual (GA e GP 100\%), discordando, assim, desses dados. A relação entre a irregularidade e a dor menstrual está associada à secreção de prostaglandinas ${ }^{15}$.

A dor ocorre principalmente na linha média e muitas vezes são descritas como cãibra no abdome inferior ou região suprapúbica, podendo irradiar em torno do abdome, na região lombar e ao longo da coxa ${ }^{1}$, assim como encontrado no presente estudo. Além disso, podem ocorrer sintomas secundários como náusea, vômito, fadiga, lombalgia, cefaleia, tontura e diarreia ${ }^{16}$, corroborando com os dados desta pesquisa. 
Um estudo avaliou 19 mulheres que mencionaram dor em baixo do ventre (100\%) e região lombar $(52,6 \%)^{15}$, o que concorda com a presente pesquisa, já que no GA $48 \%$ relataram dor na região pélvica e $8 \%$ região lombo-sacral e no GP $36 \%$ a relataram na região pélvica e $16 \%$ região lombo-sacral. A explicação mais aceita para esses achados deve-se à maior inervação do útero ocorrer nos segmentos da transição toracolombar (T10-L1) e segmentos sacrais (S2-S4) ${ }^{17}$. Petrofsky e Lee ${ }^{12}$ correlacionaram essas variáveis com a elasticidade do tecido conectivo, que estaria alterada em função da variação hormonal que ocorre nos ciclos menstruais, e encontraram diminuição do controle postural na fase de ovulação.

As principais abordagens para o tratamento da síndrome da dismenorreia primária incluem métodos farmacológicos e não farmacológicos, como psicoterapia e fisioterapia ${ }^{5}$, que oferece recursos terapêuticos tendo como finalidade a diminuição da dor de maneira prática e econômica, podendo inclusive meIhorar a qualidade de vida por meio da utilização de algumas modalidades como cinesioterapia, terapia manual e eletroterapia ${ }^{18}$.

A TENS pode ser classificada em quatro modalidades: convencional, acupuntura, em rajadas (burst) e breve-intensa. Há indícios que a TENS é efetiva tanto em alta quanto em baixa frequência, no alívio dos sintomas da dismenorreia ${ }^{19}$. Uma revisão sistemática analisou 6 estudos com a aplicação da TENS de alta frequência na dismenorreia primária e observou a eficácia desse recurso no alívio da dor, apesar da ampla variação de aplicação ${ }^{8}$, porém no presente estudo foram utilizadas alta duração de pulso e baixa frequência.

A TENS tem se mostrado um método de sucesso não invasivo no controle da dor. Seus mecanismos de ação envolvem a liberação de endorfinas no modo acupuntura, constituindo uma solução possível para mulheres que sofrem de dismenorreia e não experimentam alívio com outras formas de tratamento ${ }^{20}$. No presente estudo, observou-se redução do quadro álgico das participantes com este modo de aplicação.
Esta técnica é capaz de proporcionar analgesia por uma alteração do corpo na capacidade de percepção do estímulo doloroso, e isto fortalece a tese de que a dor na dismenorreia primária é decorrente da atividade uterina. Neste caso, ocorre a diminuição da dor pélvica, porém as contrações uterinas permanecem presentes. Este recurso terapêutico se baseia no fornecimento de corrente elétrica sobre a pele por meio de eletrodos de superfície. Seu efeito ocorre devido à estimulação de fibras sensitivas nervosas que influenciam e modulam o processo de neurocondução da dor, atuando sobre a liberação de opioides endógenos em nível medular e da hipófise ${ }^{21}$.

Um estudo clínico randomizado ${ }^{2}$ verificou que a TENS convencional (frequência $150 \mathrm{hz}$ e duração de pulso $50 \mu \mathrm{s})$ foi mais eficaz que a TENS placebo para a redução do quadro álgico de pacientes com dismenorreia primária. Apesar da utilização de parâmetros diferentes, o presente estudo também demonstrou uma redução do quadro álgico das participantes após o tratamento com a TENS.

Em uma pesquisa realizada por Machado et al. $\underline{22}$, observou-se que a TENS, com frequência $100 \mathrm{~Hz}$ e intensidade $200 \mu$ s durante 30 minutos, com os eletrodos posicionados na região pélvica anterior, proporcionou alívio rápido do quadro álgico. No presente estudo, tanto a aplicação dos eletrodos na região pélvica anterior quanto na posterior proporcionou alívio do quadro álgico das participantes. Apesar do resultado semelhante, os parâmetros usados foram diferentes.

Baldan, Freitas e Zambello ${ }^{\underline{7}}$ observaram que os eletrodos na região pélvica posterior obtiveram resultados satisfatórios por este ser um local próximo da localização do plexo hipogástrico superior (altura de L5-S1), o qual transmite os impulsos dolorosos viscerais do útero, porém encontra-se na literatura resultados satisfatórios com os eletrodos também na região pélvica anterior, estimulando os nervos sensoriais do dermátomo T12, que é raiz nervosa das fibras sensoriais uterinas, assim como observado no presente estudo. 
Para Lee et al. 9 existem evidências limitadas sobre a duração da analgesia induzida pela TENS no tratamento da dismenorreia primária. Os resultados de estudos randomizados demonstram que o quadro álgico diminui continuamente 8 e 24 horas desde 0 início da aplicação da TENS ${ }^{18}$. Nesta pesquisa, o tempo de seguimento de apenas 2 horas é considerado como uma das limitações, porém, observou-se diminuição do quadro álgico após a aplicação da técnica, assim como 2 horas após o término no GP, mas esses valores não foram estatisticamente significativos.

O modo de aplicação da TENS pode causar conflito entre os efeitos cumulativos e a tolerância. Tem sido sugerido que, para dor crônica, esse efeito cumulativo sem tolerância analgésica é alcançado apenas se a intensidade não for fixa e houver contínuo aumento, mas sem causar sensação de dor. Esse aumento é realizado à medida que a participante se acostuma com a intensidade da corrente ao longo do tempo e permite aumentar para níveis mais altos de tolerân$\mathrm{cia}^{2}{ }^{23}$, assim como neste estudo onde a intensidade foi aumentada a cada 10 minutos, a fim de evitar que as participantes se acostumassem com o estímulo aplicado.

Acredita-se que devido a uma não padronização nas metodologias, as diferenças observadas nos resultados dos estudos que abordam essa temática podem estar relacionadas ao posicionamento dos eletrodos e aos parâmetros utilizados, como frequência, duração de pulso e da aplicação, dificultando comparações entre os resultados. Não foram encontradas na literatura pesquisas que comparassem a localização da aplicação dos eletrodos na dor pélvica causada pela dismenorreia primária, no entanto, ElboimGabyzon e Kalichman $\stackrel{23}{ }$ afirmam que os eletrodos devem ser aplicados sobre a área de dor em cada ciclo menstrual. Sendo assim, pode não haver um padrão para a aplicação dos eletrodos na dismenorreia. Este estudo apresenta aplicabilidade clínica, podendo esclarecer aos fisioterapeutas sobre o uso da TENS em pacientes com dismenorreia primária.

\section{Conclusão}

Após a análise dos dados, foi possível concluir que a TENS foi eficaz para a redução do quadro álgico das participantes da pesquisa tanto no GA quanto no GP, sem diferença estatística entre os grupos. Sugere-se que outros estudos sejam realizados com número maior de participantes, tempo maior de tratamento e seguimento e a comparação de outros parâmetros, a fim de que haja padronização na utilização da TENS para alívio da sintomatologia causada pela dismenorreia primária.

\section{Contribuições das autoras}

Rodrigues AR e Almeida FO participaram da coleta e tratamento dos dados, revisão de literatura e redação. Januário PO participou da análise e interpretação dos dados e revisão crítica do texto. Cruz AT participou da concepção e desenvolvimento, planejamento, revisão de literatura, análise e interpretação, redação e revisão crítica.

\section{Conflitos de interesses}

Nenhum conflito financeiro, legal ou político envolvendo terceiros (governo, empresas e fundações privadas, etc.) foi declarado para nenhum aspecto do trabalho submetido (incluindo, mas não se limitando a subvenções e financiamentos, participação em conselho consultivo, desenho de estudo, preparação de manuscrito, análise estatística, etc.).

\section{Referências}

1. Kho KA, Shields JK. Diagnosis and Management of Primary Dysmenorrhea. JAMA. 2020;322(3):268-9. https://doi.org/10.1001/ jama.2019.16921

2. Silva BCP, Silva CKV, Pimentel TA, Souza JO, Januário PO, Cruz AT. Estimulação elétrica nervosa transcutânea no tratamento da dor pélvica causada pela dismenorreia primária. ConsSaude. 2016;15(4):650-6. https://doi.org/10.5585/conssaude.v15n4.6877

3. Acqua RD, Bendlin T. Dismenorreia [Internet]. Femina. 2015;43(6):273-6. Disponível em: http://files.bvs.br/ upload/S/0100-7254/2015/v43n6/a5327.pdf 
4. Jahangirifara $M$, Taebib $M$, Dolatianc $M$. The effect of Cinnamon on primary dysmenorrhea: A randomized, doubleblind clinical trial. Complement Ther Clin Pract. 2018;33(4):56-60. https://doi. org/10.1016/j.ctcp.2018.08.001

5. Paulino LSS, Teles A, Lordêlo P. Estimulação elétrica nervosa transcutânea na dismenorreia primária: uma revisão sistemática. Rev Pesq Fisio. 2014;4(1):47-54. http://dx.doi.org/10.17267/22382704rpf.v4i1.325

6. Barcikiwska Z, Rajkaowska-Labon E, Grzybowska ME, Hansdorfer-Korzon RH, Zorena K. Inflammatory Markers in Dysmenorrhea and Therapeutic Options. Int. J. Environ. Res. Public Health. 2020;17(4):1191. https://dx.doi. org/10.3390\%2Fijerph17041191

7. Baldan CS, Freitas CD, Zambello L. Estimulação elétrica nervosa transcutânea (TENS) alivia a dismenorreia primária: estudo clínico, controlado e randomizado [Internet]. J Health Sci Inst. 2013;31(2):193-6. Disponível em: https://www.unip.br/presencial/ comunicacao/publicacoes/ics/edicoes/2013/02_abr-jun/V31 n2 2013 p193a196.pdf

8. Oliveira MM, Cirqueira RP. Eficácia da Eletroterapia na Dismenorreia: Revisão de Literatura. Rev. Mult. Psic. 2019:13(43):448-54. https://doi.org/10.14295/idonline.v13i43.1547

9. Lee B, Hong SH, Kim K, Kang WC, No JH, Lee JR, et al. Efficacy of the device combining high-frequency transcutaneous electrical nerve stimulation and thermotherapy for relieving primary dysmenorrhea: a randomized, single-blind, placebo-controlled trial. Eur J Obs Gynecol Reprod Biol. 2015;194:58-63. https://doi. org/10.1016/j.ejogrb.2015.08.020

10. Shetty GB, Shetty B, Mooventhan A. Efficacy of Acupuncture in the Management of Primary Dysmenorrhea: A Randomized Controlled Trial. J Acupunct Meridian Stud. 2018;11(4):153-8. https://doi.org/10.1016/j.jams.2018.04.001

11. Bai HY, Bai HY, Yang ZQ. Effect of transcutaneous electrical nerve stimulation therapy for the treatment of primary dysmenorrheal. Medicine. 2017;96(36):1-4. https://doi. org/10.1097/md.0000000000007959

12. Petrofsky J, Lee H. Greater reduction of balance as a result of increased plantar fascia elasticity at ovulation during the menstrual cycle. Tohoku J Exp Med. 2015;237(3):219-26. https:// doi.org/10.1620/tjem.237.219

13. Silva FBP, Souza JO, Januário PO, Cruz AT. Prevalência Da Dismenorreia E Sua Influência Na Vida De Trabalhadoras Brasileiras [Internet]. Revista Saúde e Desenvolvimento. 2019;13(14):64-82. Disponível em: https://www.uninter.com/ revistasaude/index.php/saudeDesenvolvimento/article/ view/1017/572
14. Rodrigues AC, Gala S, Neves A, Pinto C, Meirelles C, Frutuoso $C$, et al. Dismenorreia em adolescentes e jovens adultas prevalência factores associados e limitações na vida diária. Acta Med Port. 2011;24(S2):383-92. Disponível em: https://www. actamedicaportuguesa.com/revista/index.php/amp/article/ view/1477/1063

15. Azagew AW, Kassie DG, Walle TA. Prevalence of primary dysmenorrhea, its intensity, impact and associated factors among female students' at Gondar town preparatory school, Northwest Ethiopia. BMC Womens Health. 2020;20(5):2-7. https://doi. org/10.1186/s12905-019-0873-4

16. Kannana P, Claydon LS. Some physiotherapy treatments may relieve menstrual pain in women with primary dysmenorrhea: a systematic Review. J Physiother. 2014:60(1):13-21. https://doi. org/10.1016/j.jphys.2013.12.003

17. Stallbaum JH, Silva FS, Saccol MF, Braz MM. Controle postural de mulheres com dismenorreia primária em dois momentos do ciclo menstrual. Fisioter Pesqui. 2018;25(1):74-81. http://dx.doi. org/10.1590/1809-2950/17243825012018

18. Gerzon LR, Padilha JF, Braz MM, Gasparetto A. Fisioterapia na dismenorreia primária: revisão de literatura. Rev Dor. 2014:15(4):290-5. http://dx.doi.org/10.5935/1806-0013.20140063

19. Torrilhas MC, Dresch R, Navarro YHMO, Buzanello MR, Bertolini GRF. Estimulação elétrica nervosa transcutânea na dismenorreia primária em mulheres jovens. Rev. Aten. Saúde. 2017;15(54):61-6. https://doi.org/10.13037/ras.vol15n54.4824

20. Ferreira EJ, Azanki NC. Atuação da fisioterapia na dismenorreia primária. Revista Vita et Sanitas [Internet]. 2010;4(1):57-72. Disponível em: http://fug.edu.br/revistas/index.php/VitaetSanitas/ article/view/73/62

21. Oliveira RGCQ, Silva JC, Almeida FA, Araújo RC, Pitangui ACR. TENS de alta e baixa frequência para dismenorreia primária: estudo preliminar. ConsSaude. 2012;11(1):149-58. https://doi. org/10.5585/conssaude.v11n1.2722

22. Machado AFP, Perracini MR, Rampazo EP, Driusso $P$, Liebano RE. Effects of thermotherapy and transcutaneous electrical nerve stimulation on patients with primary dysmenorrhea: A randomized, placebo-controlled, double-blind clinical trial. Complement Ther Med. 2019;47:102188. https://doi.org/10.1016/j. ctim.2019.08.022

23. Elboim-Gabyzon M, Kalichman L. Transcutaneous Electrical Nerve Stimulation (TENS) for Primary Dysmenorrhea: An Overview. International Journal of Women's Health. 2020;12:1-10. https://doi.org/10.2147/ijwh.s220523 